\title{
Supervivencia de epimastigotes de Trypanosoma cruzi en bebidas humanas contaminadas experimentalmente
}

\author{
Trujillo, A.G. ${ }^{1}$; Martínez, R.R. ${ }^{2}$; Palomino, S.J. ${ }^{1}$; Cabrera, R. ${ }^{3}$ \\ 'Escuela Prof.de Microbiol.\& Parasit., Fac.Cs.Biol., Univ.Nac.San Marcos, Lima, Perú. \\ ${ }^{2}$ Laborat. Parasit.Fauna Silv.y Zoonosis, Fac.Cs Biol., Univ.San Marcos, Lima, Perú. \\ ${ }^{3}$ Escuela de Medicina, Universidad Peruana de Ciencias Aplicadas, Lima, Perú. \\ E-mail: geraldin87@gmail.com
}

\begin{abstract}
Resumen
Trujillo, A.G.; Martínez, R.R.; Palomino, S.J.; Cabrera, R.: Supervivencia de epimas-tigotes de Trypanosoma cruzi en bebidas humanas contaminadas experimentalmente. Rev. Vet. 32: 2, 234-237, 2021. En niños de Perú se vienen reportando casos agudos de enfermedad de Chagas, asociados a focos activos o esporádicos de la cuenca amazónica, de los cuales dos casos se han vinculado epidemiológicamente al consumo de "masato" (bebida fermentada a base de yuca cocinada), planteándose una posible transmisión oral. El objetivo de esta investigación fue evaluar la supervivencia de formas epimastigotes de Trypanosoma cruzi en tres bebidas contaminadas experimentalmente, que son consumidas frecuentemente en la Amazonía peruana. Se realizaron ensayos in vitro utilizando una placa de ELISA, colocándose en cada pozo entre 100 y $200 \mu \mathrm{l}$ del filtrado de cada bebida: "chapo de maduro" (preparado a base de plátano maduro cocinado), "aguajina" (a base del fruto de aguaje) y "masato" (a base de yuca). Los jugos fueron previamente preparados (triturados, cocinados o filtrados). A cada bebida, se le adicionó $50 \mu \mathrm{l}$ del cultivo de epimastigotes de T. cruzi, mantenidas durante 7 días en medio difásico (agar sangre). La lectura se realizó a las 1, 3 y 96 horas de la contaminación experimental. La supervivencia se determinó por la ausencia del movimiento de los parásitos al examen directo. La superviviencia de epimastigotes de T. cruzi en masato fue 1 hora, en aguajina 3 horas y en chapo de maduro 96 horas. Este último podría ser un vehículo potencial para la transmisión oral de la enfermedad de Chagas en la Amazonia peruana.
\end{abstract}

Palabras clave: Trypanosoma cruzi, zumos, bebidas, técnicas in vitro.

\begin{abstract}
Trujillo, A.G.; Martínez, R.R.; Palomino, S.J.; Cabrera, R.: Survival of Trypanosoma cruzi epimastigotes in some experimentally contaminated Peruvian Amazon beverages. Rev. Vet. 32: 2, 234-237, 2021. Acute cases of Chagas disease associated with active or sporadic foci have been reported in the Amazon basin of Peru, of which two cases have been epidemiologically linked to the consumption of "masato", considering a possible oral transmission. The objective of this article is to evaluate the survival of epimastigote forms of Trypanosoma cruzi in three experimentally contaminated beverages, which are frequently consumed in the Peruvian Amazon. In vitro tests were carried out using an ELISA plate, between 100 and $200 \mu \mathrm{l}$ of the filtrate of each drink were placed in each well: "chapo de Maduro" prepared from cooked ripe banana, "aguajina" based on aguaje fruit and "masato" fermented drink based on cooked cassava. The juices were previously prepared (crushed, cooked or filtered). To each well with the drink, $50 \mu \mathrm{l}$ of the $T$. cruzi epimastigote culture, maintained for 7 days in diphasic medium (blood agar), was added. The reading was made at 1,3 and $96 \mathrm{~h}$ after the experimental contamination. Survival was determined by the absence of movement of the parasites on direct examination. Survival of T. cruzi epimastigotes in "masato" was $1 \mathrm{~h}$, "aguajina" $3 \mathrm{~h}$, and "chapo de mature" $96 \mathrm{~h}$, the latter could be a potential vehicle for oral transmission of Chagas disease in the Peruvian Amazon.
\end{abstract}

Key words: Trypanosoma cruzi, juices, beverages, in vitro techniques.

\section{INTRODUCCIÓN}

La enfermedad de Chagas es ocasionada por el parásito hemoflagelado Trypanosoma cruzi y trasmitida -principalmente- a través de la contaminación de las he- ces o de la orina de triatominos hematófagos infectados con el parásito. Sin embargo, en países donde no existe el vector, la transmisión por transfusión sanguínea, trasplante de órganos y traspaso congénito adquirió importancia ${ }^{4}$. En las zonas amazónicos, la transmisión 
oral a través de alimentos contaminados con el parásito es un problema emergente.

Se estima que a nivel global existen entre 6 y $7 \mathrm{mi}$ llones de personas infectadas con T. cruzi, principalmente en 21 países endémicos de América Latina ${ }^{25}$. Sin embargo, los años de vida ajustados por discapacidad son 7,5 veces más que la producida por la malaria ${ }^{4}$.

En los países amazónicos se ha reportado un incremento de brotes asociados a la transmisión oral de la enfermedad de chagas en Brasil, Venezuela, Colombia, Bolivia ${ }^{2}$ y Guayana Francesa ${ }^{5}$, así como también Argentina y Ecuador ${ }^{24}$. Los brotes fueron asociados a una fuente común, es decir, al consumo de bebidas y alimentos, entre ellos al jugo de açaí (fruto de una palmera) ${ }^{15}$, caña de azúcar (en Brasil) ${ }^{22}$, jugo de guayaba (en Venezuela) ${ }^{1}$ y jugo de majo (en Bolivia) ${ }^{20}$, entre otros.

Sin embargo, existen muy pocas bebidas incriminadas como vehículos para la transmisión oral de $T$. cruzi en los brotes de la enfermedad de Chagas. En un retoño en Manaos (Brasil) se aislaron cepas de T. cruzi del jugo de açaí, lográndose identificar que corresponde al linaje TcIV, tanto los que fueron identificados en los casos humanos, como en el jugo ingerido ${ }^{21}$.

Dada la importancia de evaluar la supervivencia, viabilidad, infectividad y virulencia de $T$. cruzi en los jugos o alimentos epidemiológicamente asociados en los brotes de la enfermedad de Chagas, se han realizado varios estudios. La cepa $\mathrm{Y}$ de $T$. cruzi es viable entre 4 a $12 \mathrm{~h}$ después de la contaminación del jugo de caña con el parásito y puede producir infección en ratones entre 1 y $12 \mathrm{~h}$ después de la contaminación experimental ${ }^{11,18}$.

En un reporte, las formas trypamastigotes de $T$. cruzi sobrevivieron en la pulpa de açai a temperatura ambiente hasta $144 \mathrm{~h}$ y mantuvieron su virulencia en ratones inoculados experimentalmente hasta $48 \mathrm{~h}$ después de la post-contaminación de la pulpa ${ }^{17}$. En otro estudio, en 8 de 11 muestras de frutas y hortalizas experimentalmente contaminadas con epimastigotes de $T$. cruzi, la supervivencia fue observada entre 6 y $72 \mathrm{~h}$ después de la contaminación ${ }^{3}$.

Se ha reportado un incremento de focos de transmisión activa en comunidades nativas de la Amazonia peruana, habiéndose identificado 6 casos de enfermedad de Chagas aguda (ECA) en la provincia de Datem, Marañón, Loreto y región nororiental del Perú, de los cuales dos hermanos tenían una semana de diferencia en el inicio de los síntomas, sugiriéndose que pudieron haberse infectado por vía oral a través del consumo del masato ${ }^{9}$.

También se ha postulado que el consumo del fruto de aguaje podría ser un vehículo potencial para la transmisión oral de $T$. cruzi ${ }^{10}$. Además, un estudio muy puntual identificó que las comunidades nativas del distrito de Andoas (Loreto), consumen una gran variedad de jugos y bebidas incrementando el riesgo de la transmisión oral de la enfermedad de Chagas ${ }^{7}$. En este lugar fue identificado uno de los casos de ECA ${ }^{9}$.

El objetivo de este artículo fue evaluar la supervivencia de formas epimastigotes de Trypanosoma cruzi en tres bebidas contaminadas experimentalmente, que son de consumo frecuente en la Amazonía peruana.

\section{MATERIAL Y MÉTODOS}

Se realizaron ensayos in vitro, utilizando formas epimastigotes de T. cruzi, mantenidas durante 7 días en medio difásico (agar sangre) en el Laboratorio de Parasitología de Fauna Silvestre y Zoonosis, Facultad de Ciencias Biológicas, Universidad Nacional Mayor de San Marcos, Lima.

La cepa de T. cruzi utilizada procedía del departamento de Ica, al sur del Perú. Para el ensayo se utilizaron tres bebidas de uso frecuente en la Amazonía peruana:

a) El "chapo de maduro" fue preparado a base de plátano maduro (Musa paradisiaca) cocinado en agua durante 15 min aproximadamente. Luego, fue triturado con la ayuda de un mortero de porcelana y se mezcló con el agua donde fue hervida.

b) La "aguajina" fue preparada a base del fruto de aguaje (Mauritia flexuosa), para ello la masa del fruto de aguaje fue adquirido en un mercado local de Lima.

c) El "masato" es una bebida fermentada a base de yuca cocinada (Manihot esculenta Crantz), adquirida en un mercado local de la ciudad de Lima. La masa de "aguajina" y el "masato" fueron diluidos en agua estéril.

Luego, las bebidas se filtraron individualmente utilizando un tamiz y gasa, hasta obtener una consistencia líquida. Previamente, se midió el $\mathrm{pH}$ antes del ensayo in vitro, el cual se realizó a temperatura ambiente (20$24^{\circ} \mathrm{C}$ ).

Para la evaluación del ensayo se utilizó una placa ELISA, colocando entre 100 y $200 \mu \mathrm{l}$ del filtrado de cada bebida y se adicionó $\mathbf{5 0} \mu \mathbf{l}$ del cultivo de epimastigotes de T. cruzi a cada placa. La lectura fue realizada a las 1,3 y $96 \mathrm{~h}$ de la contaminación experimental.

Para la observación de T. cruzi en las bebidas se utilizó el método directo y la supervivencia se determinó por la ausencia del movimiento de los parásitos en cada una de las muestras colocadas en los pocillos.

\section{RESULTADOS}

El ensayo in vitro fue realizado en 8 pocillos de ELISA para cada bebida. La bebida más ácida fue la "aguajina"y la bebida donde el tiempo de la supervivencia del epimastigote de T. cruzi fue más larga fue el "chapo de maduro" (Tabla 1).

\section{DISCUSIÓN}

Esta es la primera vez que se realiza un ensayo in vitro para evaluar la supervivencia de epimastigotes de T. cruzi en bebidas experimentalmente contaminadas, de consumo frecuente en la Amazonia peruana, iden- 
Tabla 1. Bebidas contaminadas experimentalmente con epimastigotes de T. cruzi: $\mathrm{pH}$ de las bebidas y supervivencia del parásito.

\begin{tabular}{|c|c|c|c|c|}
\hline \multirow{2}{*}{$\begin{array}{l}\text { bebidas contaminadas } \\
\text { experimentalmente con } \\
\text { T. cruzi }\end{array}$} & \multirow{2}{*}{$\underset{\text { (media) }}{\mathrm{pH}}$} & \multicolumn{3}{|c|}{$\begin{array}{l}\text { tiempo de supervivencia de epi- } \\
\text { mastigotes de } T \text {. cruzi en horas }\end{array}$} \\
\hline & & 1 & 3 & 96 \\
\hline "chapo de maduro" & 4,8 & $\mathrm{Si}$ & $\mathrm{Si}$ & $\mathrm{Si}$ \\
\hline "aguajina" & 3,5 & $\mathrm{Si}$ & $\mathrm{Si}$ & No \\
\hline "masato" & 3,9 & $\mathrm{Si}$ & No & No \\
\hline
\end{tabular}

tificando al "chapo de maduro", como la bebida donde existe una mayor supervivencia del parásito.

A pesar que la transmisión natural ocurre a través de la forma infectante tripomastigote metacíclico, estos resultados sugieren que el "chapo de maduro" podría ser un vehículo potencial para la transmisión oral de la enfermedad de Chagas en la Amazonia peruana.

En un estudio en Venezuela, se evaluó la supervivencia de T. cruzi en varios alimentos, entre ellos, Musa sp."plátanos". Los autores encontraron escasa cantidad de parásitos hasta las $18 \mathrm{~h}$ post-contaminación, pero los epimastigotes fueron expuestos sobre tajadas de plátanos previamente cocinados ${ }^{3}$.

En cambio, en el presente estudio se utilizó la parte líquida de la bebida "chapo de maduro", dado que es la forma natural como se consume en la Amazonia peruana. Así, los epimastigotes permanecieron móviles hasta las $96 \mathrm{~h}$ post-contaminación.

En el mismo trabajo antes mencionado, los autores, también expusieron los epimastigotes de T. cruzi en rodajas de piña, cuyo $\mathrm{pH}$ era cercano a 3,0 y no lograron observar los parásitos entre 6 a $120 \mathrm{~h}$ post-contaminación ${ }^{3}$, en cambio, en nuestro estudio en la "aguajina" que tiene una media de $\mathrm{pH}$ de 3,5 , la supervivencia fue hasta las $3 \mathrm{~h}$.

En otra investigación, en trozos de caña de azúcar contaminadas experimentalmente con las heces de triatominos infectados con la cepa $\mathrm{Y}$ de T. cruzi, luego de haberlas molido a diferentes intervalos post-contaminación $(0,1,4,6,12$ y $24 \mathrm{~h})$, al analizar el jugo fueron positivas hasta las $12 \mathrm{~h}$ post-contaminación. A pesar de ello, al inocular ratones, éstos se infectaron ${ }^{11}$.

A diferencia de la presente investigación, además del examen directo los autores usaron métodos de concentración para detectar el parásito. En una publicación previa, se había identificado la viabilidad de la cepa $Y$ de $T$. cruzi hasta $4 \mathrm{~h}$ en el jugo de caña de azúcar a temperatura ambiente, y la capacidad infectante hasta $1 \mathrm{~h}$ post-contaminación ${ }^{18}$. En el presente caso no se evaluó la supervivencia del parásito en el jugo de caña; sin embargo, un estudio exploratorio reciente puso en evidencia que algunas comunidades nativas del distrito de Andoas (departamento de Loreto), en un área de la cuenca amazónica peruana, la población consume frecuentemente el extracto de caña ${ }^{7}$.

En Brasil se evaluaron in vitro e in vivo contaminando experimentalmente con $10^{5}$ tripomastigotes las pulpas de "açai", fruto de una palmera muy consumida en Brasil y se mantuvieron a temperatura ambiente, $4^{\circ} \mathrm{C}$ y $-20^{\circ} \mathrm{C}$, dentro de diferentes períodos de incubación, encontrando los autores que en la pulpa de "açai" " mantenidas durante $48 \mathrm{~h}$ a temperatura ambiente los parásitos conservaron la virulencia en ratones inmuno suprimidos ${ }^{17}$.

Dosis de inóculo de $10^{5}$ tripomastigotes sanguíneos de T. cruzi TcI mezclados en la pulpa de "açai", aislados a partir de reservorios y vectores de Pará y Belem, en Brasil, produjeron la muerte de los ratones entre los 17 hasta los 52 días post-inoculación ${ }^{13}$

En Perú, pocos años atrás, ha sido introducida el açai (Euterpe oleracea), cuyo fruto es comestible y también se consume como jugo ${ }^{16}$. El aguaje $M$. flexuosa es otra palmera, cuyo fruto es consumido ampliamente en la Amazonia peruana. Por ello, se ha postulado que la pulpa de "aguaje" o "aguajina" podría ser un vehículo potencial para la transmisión oral de T. cruzi ${ }^{10}$.

En este estudio, la "aguajina" y el "masato" tuvieron mayor grado de acidez, lo cual podría explicarse por haber usado la forma comercial, lo cual podría ser diferente de la presentación en condiciones naturales en las zonas rurales de la Amazonia peruana. La acidez podría limitar la supervivencia de T. cruzi.

En algunas comunidades nativas de la Amazonia peruana, algunos casos confirmados de enfermedad de Chagas agudo en menores de edad, incluyendo a lactantes, tienen el antecedente de beber el "masato dulce" 9 . Éste tiene entre 1 a 3 días de fermentación ${ }^{12}$,y se espera tenga menor acidez y menor concentración alcohólica, lo que lo haría un vehículo potencial para la transmisión oral. Por ello, se sugiere evaluar la supervivencia y la capacidad infectante de T. cruzi en esas condiciones.

El incremento de reportes de casos de ECA en áreas de la cuenca Amazónica peruana ${ }^{8,9,23}$, aumenta el riesgo de la ocurrencia de brotes. Por ello, ante la notificación de un caso probable de ECA procedente de la cuenca amazónica, debe investigarse para descartar que se trate de un brote ${ }^{6}$. Además, debe promoverse la detección oportuna ${ }^{14} \mathrm{y}$ en áreas donde también es endémica la malaria, se requiere entrenar a los microscopistas para la identificación de T. cruzi en las láminas de gota gruesa y frotis ${ }^{7}$.

A pesar que en Perú no se ha comprobado la presencia de brotes por transmisión oral de T. cruzi, el personal de salud de los establecimientos de atención primaria en la cuenca amazónica, debe incluir entre sus actividades el tapado de los recipientes que se utilizan para preparar los jugos o bebidas para reducir el riesgo de la transmisión oral del parásito. Se sabe que la transmisión oral tiene mayor relevancia por su potencial epidémico y mayor severidad que la transmisión vectorial ${ }^{19}$

En este estudio no se evaluaron las formas metacíclicas de T. cruzi y tampoco se identificó el linaje del parásito; sin embargo, la identificación del "chapo de maduro" como la bebida donde las formas epimastigotes tienen mayor supervivencia es clave a la hora de in- 
vestigar un potencial brote de la enfermedad de Chagas en la Amazonia peruana.

Se concluye que el "chapo de maduro" puede ser un vehículo potencial para la transmisión oral de la enfermedad de Chagas en la Amazonia peruana, debido a la supervivencia de $96 \mathrm{~h}$ post-contaminación con epimastigotes de $T$. cruzi en esta bebida.

\section{REFERENCIAS}

1. Alarcón NB et al. 2010. Large urban outbreak of orally acquired acute Chagas disease at a school in Caracas, Venezuela. J Infect Dis 201: 9, 1308-1315.

2. Andrade DV, Gollob KJ, Dutra WO. 2014. Acute Chagas disease: new global challenges for an old neglected disease. Plos Negl Trop Dis 8: 7, 3010.

3. Añez N, Crisante G. 2008. Supervivencia de formas de cultivo de Trypanosoma cruzi en alimentos experimentalmente contaminados. Bol Mal Salud Amb 48: 1, 91-94.

4. Bern C. 2015. Chagas' disease. N Engl JMed. 373: 5, 456566.

5. Blanchet D et al. 2014. First report of a family outbreak of Chagas disease in French Guiana and post-treatment follow-up. Infect Genet Evol 28: 245-250.

6. Cabrera CR. 2006. Enfermedad de Chagas o Trypanosomiasis Americana. En: Protocolos de vigilancia epidemiológica, 2 da ed., Direcc.Gral.Epidem. Lima, Perú, 89-114.

7. Cabrera R, Valderrama Y, Meza JR. 2019. Percepción sobre la enfermedad de Chagas y el riesgo de transmisión oral en Andoas, Loreto, Perú. Rev Peru Med Exp Salud Publ. 37: 1, 174-175.

8. Cabrera R et al. 2010. Epidemiological investigation of an acute case of Chagas disease in an area of active transmission in Peruvian Amazon region. Rev Inst Med Trop Sao Paulo 52: 5, 269-272.

9. Cabrera $\mathbf{R}$ et al. 2013. New focus of active transmission of Chagas disease in indigenous populations in Peruvian A mazon basin. Rev Soc Bras Med Trop 46: 3, 367-372.

10. Cabrera R. 2006. Notas breves sobre Psammolestes tertius, Bergroth 1911 (Reduviidae: Hemiptera): un triatomino silvestre. An Fac Med (Lima) 67: 4, 345-346.

11. Cardoso AV, Lescano SA, Amato NV, Gakiya E, Santos SV. 2006. Survival of Trypanosoma cruzi in sugar cane used to prepare juice. Rev Inst Med Trop Sao Paulo 48: 5 , 287-289.

12. Daggett C. 1983. Las funciones del masato en la cultura Chayahuita. Antropológica PUCP 1: 301-310.
13. Labello BR et al. 2019. Virulence of Trypanosoma cruzi from vector and reservoir in natura açaí pulp resulting in food-borne acute Chagas disease at Pará State, Brazil. Exp Parasitol 197: 68-75.

14. Náquira C, Cabrera R. 2009. Breve reseña histórica de la enfermedad de Chagas, a cien años de su descubrimiento y situación actual en el Perú. Rev Peru MedExp Salud Públ 26: 4, 494-504.

15. Nóbrega AA et al. 2009. Oral transmission of Chagas disease by consumption of açaí palm fruit, Brazil. Emer Infec Dis 15: 4, 653-655.

16. Paniagua ZN, Bussmann RW, Macía MJ. 2017. The sociocconomic context of the use of Euterpe precatoria Mart. and E. oleracea Mart. in Bolivia and Peru. J Ethnobiol Ethnomed 13: 1, 32.

17. Passos LA et al. 2012. Sobrevivência e infectividade do Trypanosoma cruzi na polpa de açaí: estudo in vitro e in vivo. Epidemiol Serv Saúde 21: 2, 223-232.

18. Pinto PL et al. 1990. Observações sobre a viabilidade do Trypanosoma cruzi no caldo de cana. Rev Inst Med Trop Sao Paulo 32: 325-327.

19. Robertson LJ et al. 2016. Trypanosoma cruzi: Time for international recognition as a foodborne parasite. PLoSNeglTropDis 10: 6, e0004656.

20. Santalla VJ, Oporto CP, Espinoza E, Rios T, Brutus L. 2011. Primer brote reportado de la enfermedad de Chagas en la Amazonía boliviana: reporte de 14 casos agudos por transmisión oral de Trypanosoma cruzi. Biofarbo 19: 1, 52-58.

21. Santana RA et al. 2019. Oral transmission of Trypanosoma cruzi. Emerg Infect Dis 25: 1, 132-135.

22. Vargas A et al. 2018. Investigação de surto de doença de Chagas aguda na região extra-amazônica, Rio Grande do Norte, Brasil, 2016. Cad Saude Publica 34: 1 e00006517.

23. Vega $\mathbf{S}$ et al. 2006. Primer caso de enfermedad de Chagas aguda en la selva central del Perú: Investigación de colaterales, vectores y reservorios. Rev Peru Med Exp Sal Públ 23: 4, 288-292.

24. Velásquez $\mathbf{O N}$, Ramírez JD. 2020. Understanding the oral transmission of Trypanosoma cruzi as a veterinary and medical foodborne zoonosis. Res Vet Sci 132: 448-461.

25. World Health Organization. 2020. Chagas disease, also known as American trypanosomiasis. https: //www. who. int/news-room/fact-sheets/detail/chagas-disease (american trypanosomiasis) 